\title{
Two regularization methods for a class of inverse boundary value problems of elliptic type
}

Abdallah Bouzitouna' ${ }^{1}$ Nadjib Boussetila ${ }^{2 *}$ and Faouzia Rebbani ${ }^{1}$

\section{"Correspondence:}

n.boussetila@gmail.com

${ }^{2}$ Department of Mathematics, 8 Mai

1945 Guelma University, P.O. Box

401, Guelma, 24000, Algeria

Full list of author information is

available at the end of the article

\section{Springer}

\begin{abstract}
This paper deals with the problem of determining an unknown boundary condition $u(0)$ in the boundary value problem $u_{y y}(y)-A u(y)=0, u(0)=f, u(+\infty)=0$, with the aid of an extra measurement at an internal point. It is well known that such a problem is severely ill-posed, i.e., the solution does not depend continuously on the data. In order to overcome the instability of the ill-posed problem, we propose two regularization procedures: the first method is based on the spectral truncation, and the second is a version of the Kozlov-Maz'ya iteration method. Finally, some other convergence results including some explicit convergence rates are also established under a priori bound assumptions on the exact solution.
\end{abstract}

MSC: 35R25; 65J20; 35J25

Keywords: ill-posed problems; elliptic problems; cut-off spectral regularization; iterative regularization

\section{Formulation of the problem}

Throughout this paper, $H$ denotes a complex separable Hilbert space endowed with the inner product $(\cdot, \cdot)$ and the norm $\|\cdot\|, \mathcal{L}(H)$ stands for the Banach algebra of bounded linear operators on $H$.

Let $A: \mathcal{D}(A) \subset H \rightarrow H$ be a positive, self-adjoint operator with compact resolvent, so that $A$ has an orthonormal basis of eigenvectors $\left(\phi_{n}\right) \subset H$ with real eigenvalues $\left(\lambda_{n}\right) \subset \mathbb{R}_{+}$, i.e.,

$$
\begin{aligned}
& A \phi_{n}=\lambda_{n} \phi_{n}, \quad n \in \mathbb{N}^{*}, \quad\left(\phi_{i}, \phi_{j}\right)=\delta_{i j}= \begin{cases}1 & \text { if } i=j, \\
0 & \text { if } i \neq j,\end{cases} \\
& 0<v \leq \lambda_{1} \leq \lambda_{2} \leq \lambda_{3} \leq \cdots, \quad \lim _{n \rightarrow \infty} \lambda_{n}=\infty, \\
& \forall h \in H, \quad h=\sum_{n=1}^{\infty} h_{n} \phi_{n}, \quad h_{n}=\left(h, \phi_{n}\right) .
\end{aligned}
$$

In this paper, we are interested in the following inverse boundary value problem: find $(u(y), u(0))$ satisfying

$$
\begin{cases}u_{y y}-A u=0, & 0<y<\infty, \\ u(0)=f, & u(+\infty)=0,\end{cases}
$$

(o) 2013 Bouzitouna et al.; licensee Springer. This is an Open Access article distributed under the terms of the Creative Commons Attribution License (http://creativecommons.org/licenses/by/2.0), which permits unrestricted use, distribution, and reproduction in any medium, provided the original work is properly cited. 
where $f$ is the unknown boundary condition to be determined from the interior data

$$
u(b)=g \in H, \quad 0<b<\infty .
$$

This problem is an abstract version of an inverse boundary value problem, which generalizes inverse problems for second-order elliptic partial differential equations in a cylindrical domain, for example we mention the following problem.

Example 1.1 An example of (1.1) is the boundary value problem for the Laplace equation in the strip $(0, \pi) \times(0, \infty)$, where the operator $A$ is given by

$$
A=-\frac{\partial^{2}}{\partial x^{2}}, \quad \mathcal{D}(A)=H_{0}^{1}(0, \pi) \cap H^{2}(0, \pi) \subset H=L^{2}(0, \pi),
$$

which takes the form

$$
\left\{\begin{array}{l}
u_{y y}(x, y)+u_{x x}(x, y)=0, \quad x \in(0, \pi), y \in(0,+\infty), \\
u(0, y)=u(\pi, y)=0, \quad y \in(0,+\infty), \\
u(x, 0)=f(x), \quad u(x,+\infty)=0, \quad x \in[0, \pi], \\
u(x, y=b)=g(x), \quad x \in[0, \pi] .
\end{array}\right.
$$

To our knowledge, there are few papers devoted to this class of problems in the abstract setting, except for [1, 2]. In [3], the author studied a similar problem posed on a bounded interval. In this study, the algebraic invertibility of the inverse problem was established. However, the regularization aspect was not investigated.

We note here that this inverse problem was studied by Levine and Vessella [2], where the authors considered the problem of recovering $u(0)$ from the experimental data $g_{1}, \ldots, g_{n}$ associated to the internal measurements $u\left(b_{1}\right), \ldots, u\left(b_{n}\right)$, in which the temperature is measured at various depths $0<b_{1}<\cdots<b_{n}$ as approximate functions $g_{1}, \ldots, g_{n} \in H$ such that

$$
\sum_{i=1}^{n} p_{i}\left\|u\left(b_{i}\right)-g_{i}\right\|^{2} \leq \varepsilon^{2}
$$

where $p_{1}, \ldots, p_{n}$ are positive weights with $\sum_{i=1}^{n} p_{i}=1$ and $\varepsilon$ denotes the level noisy.

The regularizing strategy employed in [2] is essentially based on the Tikhonov regularization and the conditional stability estimate $\left\|u_{y}(0)\right\| \leq E$ for some $a$ priori constant $E$.

In practice, the use of $N$-measurements or the average of a series of measurements is an expensive operation, and sometimes unrealizable. Moreover, the numerical implementation of the stabilized solutions by the Tikhonov regularization method for this class of problems will be a very complex task.

For these reasons, we propose in our study a practical regularizing strategy. We show that we can recover $u(0)$ from the internal measurement $u(b)=g$ under the conditional stability estimate $\|u(0)\| \leq E$ for some a priori constant $E$. Moreover, our investigation is supplemented by numerical simulations justifying the feasibility of our approach. 


\section{Preliminaries and basic results}

In this section we present the notation and the functional setting which will be used in this paper and prepare some material which will be used in our analysis.

\subsection{Notation}

We denote by $\mathcal{C}(H)$ the set of all closed linear operators densely defined in $H$. The domain, range and kernel of a linear operator $B \in \mathcal{C}(H)$ are denoted as $\mathcal{D}(B), R(B)$ and $N(B)$; the symbols $\rho(B), \sigma(B)$ and $\sigma_{p}(B)$ are used for the resolvent set, spectrum and point spectrum of $B$, respectively. If $V$ is a closed subspace of $H$, we denote by $\Pi_{V}$ the orthogonal projection from $H$ to $V$.

For the ease of reading, we summarize some well-known facts in spectral theory.

\subsection{Spectral theorem and properties}

By the spectral theorem, for each strictly positive self-adjoint operator $B$,

$$
\begin{aligned}
& B: \mathcal{D}(B) \subset H \rightarrow H, \quad \overline{\mathcal{D}(B)}=H, \quad B=B^{*} \quad \text { and } \\
& (B u, u) \geq \gamma\|u\|^{2}, \quad \forall u \in \mathcal{D}(B)(\gamma>0),
\end{aligned}
$$

there is a unique right continuous family $\left\{E_{\lambda}, \lambda \in[\gamma, \infty[\} \subset \mathcal{L}(H)\right.$ of orthogonal projection operators such that $B=\int_{\gamma}^{\infty} \lambda d E_{\lambda}$ with

$$
\mathcal{D}(B)=\left\{v \in H: \int_{\gamma}^{\infty} \lambda^{2} d\left(E_{\lambda} v, v\right)<\infty\right\} .
$$

Theorem 2.1 [4, Theorem 6, XII.2.5, pp.1196-1198] Let $\left\{E_{\lambda}, \lambda \geq \gamma>0\right\}$ be the spectral resolution of the identity associated to $B$, and let $\Phi$ be a complex Borel function defined E-almost everywhere on the real axis. Then $\Phi(B)$ is a closed operator with dense domain. Moreover,

(i) $\mathcal{D}(\Phi(B)):=\left\{h \in H: \int_{\gamma}^{\infty}|\Phi(\lambda)|^{2} d\left(E_{\lambda} v, v\right)<\infty\right\}$,

(ii) $(\Phi(B) h, y)=\int_{\gamma}^{\infty} \Phi(\lambda) d\left(E_{\lambda} h, y\right), h \in \mathcal{D}(\Phi(B)), y \in H$,

(iii) $\|\Phi(B) h\|^{2}=\int_{\gamma}^{\infty}|\Phi(\lambda)|^{2} d\left(E_{\lambda} h, h\right), h \in \mathcal{D}(\Phi(B))$,

(iv) $\Phi(B)^{*}=\bar{\Phi}(B)$. In particular, if $\Phi$ is a real Borel function, then $\Phi(B)$ is self-adjoint.

We denote by $S(y)=e^{-y \sqrt{A}}=\sum_{n=1}^{+\infty} e^{-y \sqrt{\lambda_{n}}}\left(\cdot, \phi_{n}\right) \phi_{n} \in \mathcal{L}(H), y \geq 0$, the $C_{0}$-semigroup generated by $-\sqrt{A}$. Some basic properties of $S(y)$ are listed in the following theorem.

Theorem 2.2 (see [5], Chapter 2, Theorem 6.13, p.74) For this family of operators, we have:

1. $\|S(y)\| \leq 1, \forall y \geq 0$

2. the function $y \longmapsto S(y), y>0$, is analytic;

3. for every real $r \geq 0$ and $y>0$, the operator $S(y) \in \mathcal{L}\left(H, \mathcal{D}\left(A^{r / 2}\right)\right)$;

4. for every integer $k \geq 0$ and $y>0,\left\|S^{(k)}(y)\right\|=\left\|A^{k / 2} S(y)\right\| \leq c(k) y^{-k}$;

5. for every $h \in \mathcal{D}\left(A^{r / 2}\right), r \geq 0$, we have $S(t) A^{r / 2} h=A^{r / 2} S(y) h$.

Theorem 2.3 For $y>0, S(y)$ is self-adjoint and one-to-one operator with dense range $\left(S(y)=S(y)^{*}, \overline{\mathcal{R}(S(y))}=H\right)$. 
Proof Let $\phi_{y}:\left[0,+\infty\left[\rightarrow \mathbb{R}, s \longmapsto \phi_{y}(s)=e^{-y s}\right.\right.$. Then, by virtue of (iv) of Theorem 2.1, we can write $S(y)^{*}=\overline{\phi_{y}}(A)=\phi_{y}(A)=e^{-y \sqrt{A}}=S(y)$.

Let $h \in N\left(S\left(y_{0}\right)\right), y_{0}>0$, then $S\left(y_{0}\right) h=0$, which implies that $S(y) S\left(y_{0}\right) h=S\left(t+t_{0}\right) h=0$, $y \geq 0$. Using analyticity, we obtain that $S(y) h=0, y \geq 0$. Strong continuity at 0 now gives $h=0$. This shows that $N\left(S\left(y_{0}\right)\right)=\{0\}$.

Thanks to

$$
\overline{R\left(S\left(y_{0}\right)\right)}=N\left(S\left(y_{0}\right)\right)^{\perp}=\{0\}^{\perp}=H,
$$

we conclude that $R\left(S\left(y_{0}\right)\right)$ is dense in $H$.

Remark 2.1 For $y=b$, this theorem ensures that $S(b)$ is self-adjoint and one-to-one operator with dense range $R(S(b))$. Then we can define its inverse $S(b)^{-1}=e^{b \sqrt{A}}$, which is an unbounded self-adjoint strictly positive definite operator in $H$ with dense domain

$$
\mathcal{D}\left(S(b)^{-1}\right)=R(S(b))=\left\{h \in H:\left\|e^{b \sqrt{A}} h\right\|^{2}=\sum_{n=1}^{+\infty} e^{b \sqrt{\lambda_{n}}}\left|\left(h, \phi_{n}\right)\right|^{2}<+\infty\right\} .
$$

Let us consider the following problem: for $\xi \in H$ find $v \in \mathcal{C}^{1}(] 0,+\infty[; H) \cap \mathcal{C}([0,+\infty[$; $H) \cap \mathcal{C}(] 0,+\infty[; \mathcal{D}(A))$ such that

$$
v^{\prime}(y)+\sqrt{A} u(y)=0, \quad 0<y<+\infty, v(0)=\xi .
$$

Theorem 2.4 [6, Theorem 7.5, p.191] For any $\xi \in H$, problem (2.1) has a unique solution, given by

$$
v(y)=S(y) \xi=\sum_{n=1}^{\infty} e^{-y \sqrt{\lambda_{n}}}\left(\xi, \phi_{n}\right) \phi_{n}
$$

Moreover, for all integer $k \geq 0, v \in \mathcal{C}^{\infty}(] 0,+\infty\left[; \mathcal{D}\left(A^{k / 2}\right)\right)$. If, in addition, $\xi \in \mathcal{D}\left(A^{j / 2}\right)$, then $v \in \mathcal{C}\left(\left[0,+\infty\left[; \mathcal{D}\left(A^{j / 2}\right)\right) \cap \mathcal{C}^{j}([0,+\infty[; H)\right.\right.$ and

$$
\forall k, j \in \mathbb{N}, \quad\left\|\frac{d^{(k+j)}}{d y} v(y)\right\|=\left\|A^{k / 2} u(y)^{(j)}\right\| \leq \frac{c(k)}{y^{k}}\left\|A^{j / 2} \xi\right\| .
$$

On the other hand, Theorem 2.4 provides smoothness results with respect to $y: v \in$ $\mathcal{C}^{\infty}(] 0,+\infty[; H) \cap \mathcal{C}^{j}\left(\left[0,+\infty[; H)\right.\right.$ whenever $\xi \in \mathcal{D}\left(A^{j / 2}\right), j \in \mathbb{N}$. Under this same hypothesis, we also have smoothness in space: $v \in \mathcal{C}\left(\left[0,+\infty\left[; \mathcal{D}\left(A^{j / 2}\right)\right) \cap \mathcal{C}^{j-k}\left(\left[0,+\infty\left[; D\left(A^{k / 2}\right)\right), k \leq j\right.\right.\right.\right.$.

Here we recall a crucial theorem in the analysis of the inverse problems.

Theorem 2.5 [7, Generalized Picard theorem, p.502] Let $B: D(B) \subset H \rightarrow H$ be a selfadjoint operator and the Hilbert space $H$, and let $E_{\mu}$ be its spectral resolution of unity. Let $\theta \in \mathcal{C}(\mathbb{R}, \mathbb{R})$ and $Z(\theta):=\{t \in \mathbb{R}: \theta(t)=0\}$. We suppose that the set $Z(\theta)$ either is empty or contains isolated point only. Then the vectorial equation

$$
\theta(B) \varphi=\psi
$$


is solvable if and only if

$$
\int_{\mathbb{R}}|\theta(\lambda)|^{-2} d\left|E_{\lambda} \psi\right|^{2}<\infty .
$$

Moreover,

$$
N(\theta(B))=\{0\} \quad \Longleftrightarrow \quad \sigma_{p}(B) \cap Z(\theta)=\emptyset .
$$

On the basis $\left\{\phi_{n}\right\}$, we introduce the Hilbert scale $\left(H^{s}\right)_{s \in \mathbb{R}}\left(\right.$ resp. $\left.\left(\mathfrak{E}_{s}\right)_{s \in \mathbb{R}}\right)$ induced by $\sqrt{A}$ as follows:

$$
\begin{aligned}
& H^{s}=\left\{h \in H: \sum_{n=1}^{\infty}{\sqrt{\lambda_{n}}}^{2 s}\left|\left(h, \varphi_{n}\right)\right|^{2}<+\infty\right\}, \\
& \mathfrak{E}^{s}=\left\{h \in H: \sum_{n=1}^{\infty} e^{2 b s \sqrt{\lambda_{n}}}\left|\left(h, \varphi_{n}\right)\right|^{2}<+\infty\right\} .
\end{aligned}
$$

\subsection{Non-expansive operators}

Definition 2.1 A linear operator $M \in \mathcal{L}(H)$ is called non-expansive if

$$
\|M\| \leq 1
$$

Theorem 2.6 [8, Theorem 2.2] Let $M \in \mathcal{L}(H)$ be a positive, self-adjoint operator with $\|M\| \leq 1$. Putting $V_{0}=N(M)$ and $V_{1}=N(I-M)$, we have

$$
s-\lim _{n \rightarrow+\infty} M^{n}=\Pi_{V_{1}}, \quad s-\lim _{n \rightarrow+\infty}(I-M)^{n}=\Pi_{V_{0}}
$$

i.e.,

$$
\forall h \in H, \quad \lim _{n \rightarrow+\infty} M^{n} h=\Pi_{V_{1}} h, \quad \lim _{n \rightarrow+\infty}(I-M)^{n} h=\Pi_{V_{0}} h .
$$

For more details concerning the theory of non-expansive operators, we refer to Krasnosel'skii et al. [9, p.66].

Let use consider the operator equation

$$
S \varphi=(I-M) \varphi=\psi
$$

for non-expansive operators $M$.

Theorem 2.7 Let $M$ be a linear self-adjoint, positive and non-expansive operator on $H$. Let $\hat{\psi} \in H$ be such that equation (2.3) has a solution $\hat{\varphi}$. If 1 is not an eigenvalue of $M$, i.e., $(I-M)$ is injective $\left(V_{1}=N(I-M)=\{0\}\right)$, then the successive approximations

$$
\varphi_{n+1}=M \varphi_{n}+\hat{\psi}, \quad n=0,1,2, \ldots
$$

converge to $\hat{\varphi}$ for any initial data $\varphi_{0} \in H$. 
Proof From the hypothesis and by virtue of Theorem 2.6, we have

$$
\forall \varphi_{0} \in H, \quad M^{n} \varphi_{0} \rightarrow \Pi_{V_{1}} \varphi_{0}=\Pi_{\{0\}} \varphi_{0}=0 .
$$

By induction with respect to $n$, it is easily seen that $\varphi_{n}$ has the explicit form

$$
\begin{aligned}
\varphi_{n} & =M^{n} \varphi_{0}+\sum_{j=0}^{n-1} M^{j} \hat{\psi} \\
& =M^{n} \varphi_{0}+\left(I-M^{n}\right)(I-M)^{-1} \hat{\psi} \\
& =M^{n} \varphi_{0}+\left(I-M^{n}\right) \hat{\varphi},
\end{aligned}
$$

and (2.4) allows us to conclude that

$$
\hat{\varphi}-\varphi_{n}=M^{n}\left(\varphi_{0}-\hat{\varphi}\right) \rightarrow 0, \quad n \rightarrow \infty
$$

Remark 2.2 In many situations, some boundary value problems for partial differential equations which are ill-posed can be reduced to Fredholm operator equations of the first kind of the form $B \varphi=\psi$, where $B$ is compact, positive, and self-adjoint operator in a Hilbert space $H$. This equation can be rewritten in the following way:

$$
\varphi=(I-\omega B) \varphi+\omega \psi=L \varphi+\omega \psi
$$

where $L=(I-\omega B)$, and $\omega$ is a positive parameter satisfying $\omega<\frac{1}{\|B\|}$. It is easily seen that the operator $L$ is non-expansive and 1 is not an eigenvalue of $L$. It follows from Theorem 2.7 that the sequence $\left\{\varphi_{n}\right\}_{n=0}^{\infty}$ converges and $(I-\omega B)^{n} \zeta \rightarrow 0$ for every $\zeta \in H$ as $n \rightarrow \infty$.

\section{III-posedness and stabilization of the inverse boundary value problem}

\subsection{Cauchy problem with Dirichlet conditions}

Consider the following well-posed boundary value problem:

$$
\left\{\begin{array}{l}
v_{y y}-A v=0, \quad 0<y<\infty \\
v(0)=\xi \\
v(+\infty)=0
\end{array}\right.
$$

where $\xi$ is an $H$-valued function.

Definition $3.1[10$, p.250]

- A function $v:[0,+\infty[\rightarrow H$ is called a generalized solution to equation (3.1) if $v \in \mathbf{E}_{g}=\mathcal{C}\left(\left[0,+\infty[; H) \cap \mathcal{C}^{2}(] 0,+\infty[; H) \cap \mathcal{C}^{1}\left(\left[0,+\infty\left[; H^{-1}\right)\right.\right.\right.\right.$, and for all $\left.y \in\right] 0,+\infty[$, $u(y) \in \mathcal{D}(A)$ and obeys equation (3.1) on the same interval $] 0,+\infty[$.

- A function $v:[0,+\infty[\rightarrow H$ is called a classical solution to equation (3.1) if $v \in \mathbf{E}_{c}=\mathcal{C}^{1}\left(\left[0,+\infty[; H) \cap \mathcal{C}^{2}(] 0,+\infty[; H)\right.\right.$, and for all $\left.y \in\right] 0,+\infty[, u(y) \in \mathcal{D}(A)$ and obeys equation (3.1) on the same interval $] 0,+\infty[$. 
Theorem 3.1 Problem (3.1) admits a unique generalized (resp. classical) solution if and only if $\xi \in H$ (resp. $\left.\xi \in H^{1}\right)$.

Proof By using the Fourier expansion and the given Dirichlet boundary conditions

$$
\begin{aligned}
& v(y)=\sum_{n=1}^{+\infty} v_{n}(y) \phi_{n}, \\
& v(0)=\sum_{n=1}^{+\infty} v_{n}(0) \phi_{n}=\xi=\sum_{n=1}^{+\infty} \xi_{n} \phi_{n}, \\
& v(+\infty)=\sum_{n=1}^{+\infty} v_{n}(+\infty) \phi_{n}=0,
\end{aligned}
$$

we obtain

$$
\left\{\begin{array}{l}
v_{n}^{\prime \prime}-\lambda_{n} v_{n}(y)=0, \quad 0<y<\infty \\
v_{n}(0)=\xi_{n} \\
v_{n}(+\infty)=0
\end{array}\right.
$$

This differential equation admits two linearly independent fundamental solutions

$$
\varphi_{n}^{+}(y)=e^{+y \sqrt{\lambda_{n}}}, \quad \varphi_{n}^{-}(y)=e^{-y \sqrt{\lambda_{n}}} .
$$

Thus, its general solution can be written as

$$
v_{n}(y)=c_{n}^{+} e^{+y \sqrt{\lambda_{n}}}+c_{n}^{-} e^{-y \sqrt{\lambda_{n}}}, \quad c_{n}^{+}, c_{n}^{-} \in \mathbb{R} .
$$

Applying $v_{n}(+\infty)=0$ and $v_{n}(0)=\xi_{n}$ yields $c_{n}^{+}=0$ and $c_{n}^{-}=\xi_{n}$. Finally, the solution of (3.2) is

$$
\nu(y)=S(y) \xi=e^{-y \sqrt{A}} \xi=\sum_{n=1}^{+\infty} e^{-y \sqrt{\lambda_{n}}} \xi_{n} \phi_{n}, \quad \xi_{n}=\left(\xi, \phi_{n}\right) .
$$

Remark 3.1 It is easy to check that the expression (3.3) solves the problem

$$
\left.u^{\prime}(y)+\sqrt{A} u(y)=0, \quad y \in\right] 0,+\infty[, \quad u(0)=\xi .
$$

If $\xi \in H$ (resp. $\xi \in H^{1}$ ), by virtue of Theorem 2.4 and Remark 3.1, we easily check the inclusion $v \in \mathbf{E}_{g}$ (resp. $v \in \mathbf{E}_{c}$ ) and $v(y) \in D(A)$ for $\left.y \in\right] 0,+\infty[$.

\subsection{Inverse boundary value problem}

Our inverse problem is to determine $v(0)=f$ from the supplementary condition $v(b)=g$, then we get

$$
\nu(b)=\sum_{n=1}^{+\infty} e^{-b \sqrt{\lambda_{n}}} f_{n} \phi_{n}=g=\sum_{n=1}^{+\infty} g_{n} \phi_{n} .
$$


We define

$$
K=S(b): H \rightarrow H, \quad h \longmapsto K h=\sum_{n=1}^{+\infty} e^{-b \sqrt{\lambda_{n}}} h_{n} \phi_{n} .
$$

The operator equation (3.5) is the main instrument in investigating problem (3.4). More precisely, we want to study the following properties:

1. Injectivity of $K$ (identifiability);

2. Continuity of $K$ and the existence of its inverse (stability);

3. The range of $K$.

It is easy to see that $K$ is a linear compact self-adjoint operator with the singular values $\left(\sigma_{k}=e^{-b \sqrt{\lambda_{k}}}\right)_{k=1}^{+\infty}$, and by virtue of Remark 2.1, we have

1. $N(K)=\{0\}$,

2. $R(K)=\left\{h \in H:\left\|e^{b \sqrt{A}} h\right\|^{2}=\sum_{n=1}^{+\infty} e^{2 b \sqrt{\lambda_{n}}}\left|\left(h, \phi_{n}\right)\right|^{2}<+\infty\right\}$,

3. $\overline{R(K)}=H$.

Now, to conclude the solvability of problem (3.4) it is enough to apply Theorem 2.5.

Corollary 3.1 The inverse problem (3.4) is uniquely solvable if and only if

$$
u(b)=g \in R(K)=\left\{h \in H: \sum_{n=1}^{+\infty} e^{2 b \sqrt{\lambda_{n}}}\left|\left(h, \phi_{n}\right)\right|^{2}<+\infty\right\} .
$$

In this case, we have

$$
f=u(0)=K^{-1} g=\sum_{n=1}^{+\infty} e^{b \sqrt{\lambda_{n}}} g_{n} \phi_{n} .
$$

In other words, the solution $f$ of the inverse problem is obtained from the data $g$ via the unbounded operator $\mathbf{L}=K^{-1}$ defined on functions $g$ in the subspace

$$
D(\mathbf{L})=\left\{g \in H: \sum_{n=1}^{+\infty} e^{2 b \sqrt{\lambda_{n}}}\left|\left(g, \phi_{n}\right)\right|^{2}<+\infty, g_{n}=\left(g, \phi_{n}\right)\right\} .
$$

Corollary 3.2 Problem (1.1)-(1.2) admits a unique solution $u \in C([0,+\infty[; H)$ if and only if

$$
u(0) \in H \quad \Longleftrightarrow \quad g \in R(K)=\left\{h \in H: \sum_{n=1}^{+\infty} e^{b \sqrt{\lambda_{n}}}\left|\left(h, \phi_{n}\right)\right|^{2}<+\infty\right\} .
$$

In this case, we have

$$
u(y)=e^{(b-y) \sqrt{A}} g=\sum_{n=1}^{+\infty} e^{(b-y) \sqrt{\lambda_{n}}} g_{n} \phi_{n} .
$$


From this representation, we see that:

- $u(y)$ is stable in the interval $\left[b,+\infty\left[\left(\sup _{y \in[b,+\infty}\|u(y)\| \leq\|g\|\right)\right.\right.$;

- $u$ is unstable in $\left[0, b\left[\right.\right.$. This follows from the high-frequency $\omega_{n}=e^{(b-y) \sqrt{\lambda_{n}}} \rightarrow+\infty$, $n \rightarrow+\infty$.

\subsection{Regularization by truncation method and error estimates}

A natural way to stabilize the problem is to eliminate all the components of large $n$ from the solution and instead consider (3.7) only for $n \leq N$.

Definition 3.2 For $N>0$, the regularized solution of problem (1.1)-(1.2) is given by

$$
\begin{aligned}
& f_{N}=\sum_{n \leq N} e^{b \sqrt{\lambda_{n}}} g_{n} \phi_{n}, \quad g_{n}=\left(g, \phi_{n}\right) \\
& u_{N}(y)=\sum_{n \leq N} e^{(b-y) \sqrt{\lambda_{n}}} g_{n} \phi_{n}, \quad g_{n}=\left(g, \phi_{n}\right) .
\end{aligned}
$$

Remark 3.2 If the parameter $N$ is large, $f_{N}$ is close to the exact solution $f$. On the other hand, if the parameter $N$ is fixed, $f_{N}$ is bounded. So, the positive integer $N$ plays the role of regularization parameter.

Remark 3.3 In view of

$$
\left\|u(y)-u_{N}(y)\right\|=\left\|S(y)\left(f-f_{N}\right)\right\| \leq\left\|\left(f-f_{N}\right)\right\| \quad \Longrightarrow \quad\left\|u-u_{N}\right\|_{\infty} \leq\left\|\left(f-f_{N}\right)\right\|,
$$

and if $g \in \mathfrak{E}^{1}$, i.e., $\sum_{n=1}^{\infty} e^{2 b \sqrt{\lambda_{n}}}\left|\left(g, \phi_{n}\right)\right|^{2}<\infty$, then

$$
\left\|f-f_{N}\right\| \rightarrow 0, \quad N \rightarrow \infty
$$

implies

$$
\left\|u-u_{N}\right\|_{\infty}=\sup _{y \in[0,+\infty[}\left\|u(y)-u_{N}(y)\right\| \rightarrow 0, \quad N \rightarrow \infty
$$

Since the data $g$ are based on (physical) observations and are not known with complete accuracy, we assume that $g$ and $g^{\delta}$ satisfy $\left\|g-g^{\delta}\right\| \leq \delta$, where $g^{\delta}$ denotes the measured data and $\delta$ denotes the level noisy.

Let $\left(f_{N}^{\delta}, u_{N}^{\delta}\right)$ denote the regularized solution of problem (1.1), (1.2) with measured data $g_{\delta}$ :

$$
\begin{aligned}
& f_{N}^{\delta}=\sum_{n \leq N} e^{b \sqrt{\lambda_{n}}} g_{n}^{\delta} \phi_{n}, \quad g_{n}^{\delta}=\left(g^{\delta}, \phi_{n}\right), \\
& u_{N}^{\delta}(y)=\sum_{n \leq N} e^{(b-y) \sqrt{\lambda_{n}}} g_{n}^{\delta} \phi_{n}, \quad g_{n}^{\delta}=\left(g^{\delta}, \phi_{n}\right) .
\end{aligned}
$$

As usual, in order to obtain convergence rate, we assume that there exists an $a$ priori bound for problem (1.2)

$$
\left\|A^{r / 2} f\right\|^{2} \leq E^{2}<+\infty \Longleftrightarrow \sum_{n=1}^{+\infty} \lambda_{n}^{r} e^{2 b \sqrt{\lambda_{n}}}\left|g_{n}\right|^{2} \leq E^{2}
$$

where $E>0$ is a given constant. 
Remark 3.4 For given two exact conditions $g_{1}$ and $g_{2}$, let $f_{1, N}$ and $f_{2, N}$ be the corresponding regularized solutions, respectively. Then

$$
\left\|f_{2, N}-f_{1, N}\right\|^{2}=\sum_{n \leq N} e^{2 b \sqrt{\lambda_{n}}}\left|\left(g_{2}-g_{1}\right)_{k}\right|^{2} \leq e^{2 b \sqrt{\lambda_{N}}}\left\|g_{2}-g_{1}\right\|^{2} .
$$

The main theorem of this method is as follows.

Theorem 3.2 Let $f_{N}^{\delta}$ be the regularized solution given by (3.11), and let $f$ be the exact solution given by (3.7). If $\left\|A^{r / 2} f\right\| \leq E, r>0$ and if we choose $\sqrt{\lambda_{N}} \approx \frac{\theta}{b} \log \left(\frac{1}{\delta}\right), 0<\theta<1$, then we have the error bound

$$
\left\|f-f_{N}^{\delta}\right\| \leq\left(\frac{b}{\theta}\right)^{r}\left(\frac{1}{\log (1 / \delta)}\right)^{r} E+\delta^{1-\theta} .
$$

Proof From direct computations, we have

$$
\begin{aligned}
& \Delta_{1}=\left\|f_{N}-f_{N}^{\delta}\right\| \leq e^{b \sqrt{\lambda_{N}}}\left\|g-g^{\delta}\right\| \leq e^{b \sqrt{\lambda_{N}}} \delta, \\
& \Delta_{2}^{2}=\left\|f-f_{N}\right\|^{2}=\sum_{n=N+1}^{+\infty} e^{2 b \sqrt{\lambda_{n}}}\left|g_{n}\right|^{2} \\
& =\sum_{n=N+1}^{+\infty} \frac{1}{{\sqrt{\lambda_{n}}}^{2 r}}{\sqrt{\lambda_{n}}}^{2 r} e^{2 b \sqrt{\lambda_{n}}}\left|g_{n}\right|^{2} \\
& \leq \frac{1}{\sqrt{\lambda_{N+1}}}{ }^{2 r} \sum_{n=N+1}^{+\infty}{\sqrt{\lambda_{n}}}^{2 r} e^{2 b \sqrt{\lambda_{n}}}\left|g_{n}\right|^{2} \\
& \leq\left(\frac{1}{\sqrt{\lambda_{N}}}\right)^{2 r} E^{2} \text {. }
\end{aligned}
$$

Using the triangle inequality

$$
\left\|f-f_{N}^{\delta}\right\| \leq\left\|f-f_{N}\right\|+\left\|f_{N}-f_{N}^{\delta}\right\|=\Delta_{1}+\Delta_{2}
$$

we obtain

$$
\left\|f-f_{N}^{\delta}\right\| \leq\left(\frac{1}{\sqrt{\lambda_{N}}}\right)^{r} E+e^{b \sqrt{\lambda_{N}}} \delta .
$$

By choosing $\sqrt{\lambda_{N}}=\frac{\theta}{b} \log \left(\frac{1}{\delta}\right), 0<\theta<1$, we obtain

$$
\left\|f-f_{N}^{\delta}\right\| \leq\left(\frac{b}{\theta}\right)^{r}\left(\frac{1}{\log (1 / \delta)}\right)^{r} E+\delta^{1-\theta} .
$$

Finally, from (3.4) and (3.15), we deduce the following corollary.

Corollary 3.3 Let $u_{N}^{\delta}$ be the regularized solution given by (3.12), and let $u$ be the exact solution given by (3.8). If $\left\|A^{r / 2} f\right\| \leq E, r>0$ and if we choose $\sqrt{\lambda_{N}}=\frac{\theta}{b} \log \left(\frac{1}{\delta}\right), 0<\theta<1$, then we have the error bound

$$
\left\|u-u_{N}^{\delta}\right\|_{\infty}=\sup _{y \in[0,+\infty[}\left\|u(y)-u_{N}^{\delta}(y)\right\| \leq\left\|f-f_{N}^{\delta}\right\| \leq\left(\frac{b}{\theta}\right)^{r}\left(\frac{1}{\log (1 / \delta)}\right)^{r} E+\delta^{1-\theta} .
$$




\section{Regularization by the Kozlov-Maz'ya iteration method and error estimates}

In $[11,12]$ Kozlov and Maz'ya proposed an alternating iterative method to solve boundary value problems for general strongly elliptic and formally self-adjoint systems. After that, the idea of this method has been successfully used for solving various classes of ill-posed (elliptic, parabolic and hyperbolic) problems; see, e.g., [13-15].

In this section we extend this method to our ill-posed problem.

\subsection{Description of the method}

The iterative algorithm for solving the inverse problem (1.1)-(1.2) starts by letting $f_{0} \in H$ be arbitrary. The first approximation $u^{0}(y)$ is the solution to the direct problem

$$
\left\{\begin{array}{l}
u_{y y}^{0}-A u^{0}=0, \quad 0<y<\infty \\
u^{0}(0)=f_{0} \\
u(+\infty)=0
\end{array}\right.
$$

If the pair $\left(u^{k}, f_{k}\right)$ has been constructed, let

$$
(\mathrm{P})_{k+1}: \quad f_{k+1}=f_{k}-\omega\left(u^{k}(b)-f\right),
$$

where $\omega$ is such that

$$
0<\omega<\frac{1}{\|K\|}=e^{b \sqrt{\lambda_{1}}}, \quad\|K\|=\sup _{n} e^{-b \sqrt{\lambda_{n}}}=e^{-b \sqrt{\lambda_{1}}}<1 .
$$

Finally, we get $u^{k+1}$ by solving the problem

$$
\left\{\begin{array}{l}
u_{y y}^{k+1}-A u^{k+1}=0, \quad 0<y<\infty \\
u^{k+1}(0)=f_{k+1} \\
u^{k+1}(+\infty)=0
\end{array}\right.
$$

We set $G=(I-\omega K)$. If we iterate backwards in $(\mathrm{P})_{k+1}$, we obtain

$$
f_{k}=G^{k} f_{0}+\omega \sum_{i=0}^{k-1} G^{i} g=G^{k} f_{0}+\left(I-G^{k}\right) K^{-1} g=G^{k} f_{0}+f-G^{k} f .
$$

This implies that

$$
f_{k}-f=G^{k}\left(f_{0}-f\right), \quad u^{k}(y)-u(y)=S(y) G^{k}\left(f_{0}-f\right) .
$$

Proposition 4.1 The operator $G=(I-\omega K)$ is self-adjoint and non-expansive on H. Moreover, it has not 1 as eigenvalue.

Proof The self-adjointness follows from the definition of $G$ (see Theorem 2.1). Since the inequality $0<1-\omega e^{-b \sqrt{\lambda}}<1$ for $\lambda \in \sigma(A)$, we have $\left.\sigma_{p}(G) \subset\right] 0,1[$, then 1 is not an eigenvalue of $G$. 
In general, the exact solution $u(0)=f \in H$ is required to satisfy the so-called source condition; otherwise, the convergence of the regularization method approximating the problem can be arbitrarily slow. Since our problem is exponentially ill-posed (the eigenvalues $s_{n}=e^{-b \sqrt{\lambda_{n}}}$ of $K$ converge exponentially to 0 ), it is well known in this case [16, 17] that the best choice to accelerate the convergence of the regularization method is to use logarithmic-type source conditions, i.e.,

$$
\left(f_{0}-f\right)=\Psi_{\beta}(\omega K) \xi, \quad \xi \in H,\|\xi\| \leq E
$$

where

$$
\Psi_{\beta}(t)= \begin{cases}\ln \left(\frac{e}{t}\right)^{-\beta}, & 0<t \leq 1, \\ 0, & t=0,\end{cases}
$$

with $\beta>0$.

Remark 4.1 [16, p.34] The logarithmic source condition $\zeta=\left(f_{0}-f\right) \in R\left(\Psi_{\beta}(\omega K)\right)$ is equivalent to the inclusion $\zeta \in R\left(A^{-\beta / 2}\right)=D\left(A^{\beta / 2}\right)$.

Proof The proof is based on the following equivalence:

$$
\sum_{k=1}^{\infty}\left(\ln \left(\frac{e}{\omega}\right)+\sqrt{\lambda_{n}}\right)^{2 \beta}<+\infty \Longleftrightarrow \sum_{k=1}^{\infty}\left(\sqrt{\lambda_{n}}\right)^{2 \beta}<+\infty .
$$

Lemma 4.1 [18, Appendix, Lemma A.1] Let $\beta>0$ and $k \in \mathbb{N}, k \geq 2$. Then the real-valued function $\tau(t)=(1-t)^{k} \ln \left(\frac{e}{t}\right)^{-\beta}$ defined on $[0,1]$ satisfies

$$
\tau(t) \leq C \ln (k)^{-\beta} .
$$

Remark 4.2 Let $k \in \mathbb{N}^{*}$. Then the real-valued function $\varrho(t)=1-(1-t)^{k}$ defined on $[0,1]$ satisfies

$$
\varrho(t) \leq k t
$$

Proof Using the mean value theorem, we can write

$$
\varrho(t)-\varrho(0)=(t-0) \varrho^{\prime}(\hat{t}), \quad 0<\hat{t}<t,
$$

then

$$
\varrho(t)=t k(1-\hat{t})^{k-1} \leq k t
$$

Let us consider the following real-valued functions:

$$
\begin{aligned}
& Q(\lambda)=\left(1-\omega e^{-b \sqrt{\lambda}}\right)^{k} \ln \left(\frac{e}{\omega e^{-b \sqrt{\lambda}}}\right)^{-\beta}, \quad \lambda \in\left[\lambda_{1},+\infty[,\right. \\
& P(\lambda)=\omega \sum_{i=0}^{k-1}\left(1-\omega e^{-b \sqrt{\lambda}}\right)^{i}=\omega \frac{1-\left(1-\omega e^{-b \sqrt{\lambda}}\right)^{k}}{\omega e^{-b \sqrt{\lambda}}}, \quad \lambda \in\left[\lambda_{1},+\infty[.\right.
\end{aligned}
$$


Using the change of variables $t=\vartheta(\lambda)=\omega e^{-b \sqrt{\lambda}}$, we obtain

$$
\begin{aligned}
& \hat{Q}(t)=Q\left(\vartheta^{-1}(t)\right)=(1-t)^{k} \ln \left(\frac{e}{t}\right)^{-\beta}, \quad t \in[0,1], \\
& \hat{P}(t)=P\left(\vartheta^{-1}(t)\right)= \begin{cases}\omega \frac{1-(1-t)^{k}}{t}, & t \in] 0,1], \\
\omega k, & t=0 .\end{cases}
\end{aligned}
$$

Now we are in a position to state the main result of this method.

Theorem 4.1 Let $g \in \mathfrak{E}^{1}$ and $\omega$ satisfy $0<\omega<e^{b \sqrt{\lambda_{1}}}$, let $f_{0}$ be an arbitrary element for the iterative procedure suggested above, and let $u^{k}$ be the kth approximate solution. Then we have

$$
\sup _{y \in[0,+\infty[}\left\|u(y)-u^{k}(y)\right\| \rightarrow 0, \quad k \rightarrow \infty
$$

Moreover, if $\left(f_{0}-f\right) \in H^{\beta}(\beta>0)$, i.e., $\left(f_{0}-f\right)=\Psi_{\beta}(\omega K) \xi, \xi \in H,\|\xi\| \leq E$, then the rate of convergence of the method is given by

$$
\sup _{y \in[0,+\infty[}\left\|u(y)-u^{k}(y)\right\| \leq C E\left(\frac{1}{\ln (k)}\right)^{\beta}, \quad k \geq 2 .
$$

Proof By virtue of Proposition 4.1 and Theorem 2.7, it follows immediately

$$
\sup _{y \in[0,+\infty[}\left\|u(y)-u^{k}(y)\right\| \leq\left\|G^{k}\left(f_{0}-f\right)\right\| \rightarrow 0, \quad k \rightarrow \infty .
$$

We have

$$
\begin{aligned}
\left\|u(y)-u^{k}(y)\right\|^{2} & =\left\|S(y) G^{k}\left(f_{0}-f\right)\right\|^{2} \\
& \leq\left\|G^{k}\left(f_{0}-f\right)\right\|^{2}=\sum_{n=1}^{\infty} Q\left(\lambda_{n}\right)^{2}\left\|\left(\xi, \phi_{n}\right)\right\|^{2} \\
& \leq\left(\sup _{t \in[0,1]} \hat{Q}(t)\right)^{2}\|\xi\|^{2} \leq\left(\sup _{t \in[0,1]} \hat{Q}(t)\right)^{2} E^{2},
\end{aligned}
$$

and by virtue of Lemma 4.1 (estimate (4.7)), we conclude the desired estimate.

Theorem 4.2 Let $g \in \mathfrak{E}^{1}$ and $\omega$ satisfy $0<\omega<e^{b \sqrt{\lambda_{1}}}$, let $f_{0}$ be an arbitrary element for the iterative procedure suggested above, and let $u^{k}\left(\right.$ resp. $\left.u_{k}^{\delta}\right)$ be the kth approximate solution for the exact data $g$ (resp. for the inexact data $g^{\delta}$ ) such that $\left\|g-g^{\delta}\right\| \leq \delta$. Then, under condition (4.6), the following inequality holds:

$$
\sup _{y \in[0,+\infty[}\left\|u(y)-u_{\delta}^{k}(y)\right\| \leq C E\left(\frac{1}{\ln (k)}\right)^{\beta}+\varepsilon(k) \delta,
$$

where $\varepsilon(k)=\left\|\omega \sum_{i=0}^{k-1}(I-\omega K)^{i}\right\| \leq k \omega$. 
Proof Using (4.4) and the triangle inequality, we can write

$$
\begin{aligned}
& f^{k}=G^{k} f_{0}+\omega \sum_{i=0}^{k-1} G^{i} g, u_{k}(y)=S(y) f^{k}, \\
& f_{\delta}^{k}=G^{k} f_{0}+\omega \sum_{i=0}^{k-1} G^{i} g^{\delta}, u_{k}^{\delta}(y)=S(y) f_{\delta}^{k}, \\
& \left\|u(y)-u_{\delta}^{k}(y)\right\|=\left\|\left(u(y)-u^{k}(y)\right)+\left(u^{k}(y)-u_{\delta}^{k}(y)\right)\right\| \leq \Delta_{1}+\Delta_{2},
\end{aligned}
$$

where

$$
\Delta_{1}=\left\|u(y)-u^{k}(y)\right\| \leq\left\|u(y)-u^{k}(y)\right\|_{\infty} \leq C E\left(\frac{1}{\ln (k)}\right)^{\beta}, \quad k \geq 2,
$$

and

$$
\begin{aligned}
\Delta_{2} & =\left\|u^{k}(y)-u_{\delta}^{k}(y)\right\|=\left\|S(y)\left(f^{k}-f_{\delta}^{k}\right)\right\|=\left\|\omega S(y) \sum_{i=0}^{k-1} G^{i}\left(g-g^{\delta}\right)\right\| \\
& \leq\left\|\omega \sum_{i=0}^{k-1} G^{i}\left(g-g^{\delta}\right)\right\| \leq\left\|\omega \sum_{i=0}^{k-1} G^{i}\right\| \delta=\hat{\Delta}_{2} .
\end{aligned}
$$

By using inequality (4.8), the quantity $\hat{\Delta}_{2}$ can be estimated as follows:

$$
\hat{\Delta}_{2} \leq \omega k \delta
$$

Combining (4.13) and (4.14) and taking the supremum with respect to $y \in[0,+\infty[$ of $\left\|u(y)-u_{\delta}^{k}(y)\right\|$, we obtain the desired bound.

Remark 4.3 Choosing $k=k(\delta)$ such that $\omega k \delta \rightarrow+\infty$ as $\delta \rightarrow 0$, we obtain

$$
\sup _{y \in[0,+\infty[}\left\|u^{k}(y)-u_{\delta}^{k}(y)\right\| \rightarrow 0 \quad \text { as } k \rightarrow+\infty .
$$

\section{Numerical results}

In this section we give a two-dimensional numerical test to show the feasibility and efficiency of the proposed methods. Numerical experiments were carried out using MATLAB.

We consider the following inverse problem:

$$
\left\{\begin{array}{l}
u_{y y}(x, y)+u_{x x}(x, y)=0, \quad x \in(0, \pi), y \in(0,+\infty), \\
u(0, y)=u(\pi, y)=0, \quad y \in(0,+\infty), \\
u(x, 0)=f(x), \quad u(x,+\infty)=0, \quad x \in[0, \pi],
\end{array}\right.
$$

where $f(x)$ is the unknown source and $u(x, 1)=g(x)$ is the supplementary condition.

It is easy to check that the operator

$$
A=-\frac{\partial^{2}}{\partial x^{2}}, \quad \mathcal{D}(A)=H_{0}^{1}(0, \pi) \cap H^{2}(0, \pi) \subset H=L^{2}(0, \pi)
$$

is positive, self-adjoint with compact resolvent ( $A$ is diagonalizable). 
The eigenpairs $\left(\lambda_{n}, \phi_{n}\right)$ of $A$ are

$$
\lambda_{n}=n^{2}, \quad \phi_{n}(x)=\sqrt{\frac{2}{\pi}} \sin (n x), \quad n \in \mathbb{N}^{*} .
$$

In this case, formula (3.7) takes the form

$$
\begin{aligned}
f(x) & =u(x, 0)=K^{-1} g(x) \\
& =\frac{2}{\pi} \sum_{n=1}^{+\infty} e^{n}\left(\int_{0}^{\pi} g(x) \sin (n x) d x\right) \sin (n x) .
\end{aligned}
$$

\section{Truncation method}

We use trapezoid's rule to approach the integral and do an approximate truncation for the series by choosing the sum of the front $M+1$ terms. After considering an equidistant grid $0=x_{1}<\cdots<x_{M+1}=\pi, x_{j}=(j-1) h=(j-1) \frac{\pi}{M}, j=1 \cdots(M+1)$, we get

$$
\begin{aligned}
& f\left(x_{j}\right)=\frac{2}{\pi} \sum_{i=1}^{M+1} \sum_{n=1}^{+\infty} e^{n}\left(h g\left(x_{i}\right) \sin \left(n x_{i}\right)\right) \sin \left(n x_{j}\right), \\
& f_{N}\left(x_{j}\right)=\frac{2}{\pi} \sum_{i=1}^{M+1} \sum_{n=1}^{N} e^{n}\left(h g\left(x_{i}\right) \sin \left(n x_{i}\right)\right) \sin \left(n x_{j}\right), \\
& f_{N}^{\delta}\left(x_{j}\right)=\frac{2}{\pi} \sum_{i=1}^{M+1} \sum_{n=1}^{N} e^{n}\left(h g^{\delta}\left(x_{i}\right) \sin \left(n x_{i}\right)\right) \sin \left(n x_{j}\right) .
\end{aligned}
$$

In the following, we consider an example which has an exact expression of solutions $(u(x, y), f(x))$.

\section{Example}

If $u(x, 0)=\sqrt{\frac{2}{\pi}} e \sin (x)$, then the function $u(x, y)=\sqrt{\frac{2}{\pi}} e^{1-y} \sin (x)$ is the exact solution of problem (5.1). Consequently, the data function is $g(x)=u(x, 1)=\sqrt{\frac{2}{\pi}} \sin (x)$.

Adding a random distributed perturbation (obtained by the Matlab command randn) to each data function, we obtain the vector $g^{\delta}$ :

$$
g^{\delta}=g+\varepsilon \operatorname{randn}(\operatorname{size}(g))
$$

where $\varepsilon$ indicates the noise level of the measurement data and the function ' $\operatorname{randn}(\cdot)^{\prime}$ ' generates arrays of random numbers whose elements are normally distributed with mean 0 , variance $\sigma^{2}=1$, and standard deviation $\sigma=1$. ' $\operatorname{randn}(\operatorname{size}(g))^{\prime}$ ' returns an array of random entries that is the same size as $g$. The bound on the measurement error $\delta$ can be measured in the sense of Root Mean Square Error (RMSE) according to

$$
\delta=\left\|g^{\delta}-g\right\|_{*}=\left(\frac{1}{M+1} \sum_{i=1}^{M+1}\left(g\left(x_{i}\right)-g^{\delta}\left(x_{i}\right)\right)^{2}\right)^{1 / 2} .
$$


Using $g^{\delta}$ as a data function, we obtain the computed approximation $f_{N}^{\delta}$ by (5.5). The relative error $E_{r}(f)$ is given by

$$
E_{r}(f)=\frac{\left\|f_{N}^{\delta}-f\right\|_{*}}{\|f\|_{*}}
$$

\section{Kozlov-Maz'ya iteration method}

By using the central difference with step length $h=\frac{\pi}{N+1}$ to approximate the first derivative $u_{x}$ and the second derivative $u_{x x}$, we can get the following semi-discrete problem (ordinary differential equation):

$$
\left\{\begin{array}{l}
u_{y y}\left(x_{i}, y\right)-\mathbb{A}_{h}\left(x_{i}, y\right)=0, \quad x_{i}=i h, i=1, \ldots, N, y \in(0,+\infty), \\
u\left(x_{0}=0, y\right)=u\left(x_{N+1}=\pi, y\right)=0, \quad y \in(0,+\infty), \\
u\left(x_{i}, 0\right)=f\left(x_{i}\right), \quad u\left(x_{i},+\infty\right)=0, \quad x_{i}=i h, i=1, \ldots, N,
\end{array}\right.
$$

where $\mathbb{A}_{h}$ is the discretization matrix stemming from the operator $A=-\frac{d^{2}}{d x^{2}}$ :

$$
\mathbb{A}_{h}=\frac{1}{h^{2}} \operatorname{Tridiag}(-1,2,-1) \in \mathcal{M}_{N}(\mathbb{R})
$$

is a symmetric, positive definite matrix. We assume that it is fine enough so that the discretization errors are small compared to the uncertainty $\delta$ of the data; this means that $\mathbb{A}_{h}$ is a good approximation of the differential operator $A=-\frac{d^{2}}{d x^{2}}$, whose unboundedness is reflected in a large norm of $\mathbb{A}_{h}$ (see [19, p.5]). The eigenpairs $\left(\mu_{k}, e_{k}\right)$ of $\mathbb{A}_{h}$ are given by

$$
\mu_{k}=4\left(\frac{N+1}{\pi}\right)^{2} \sin ^{2}\left(\frac{k \pi}{2(N+1)}\right), \quad e_{k}=\left(\sin \left(\frac{j k \pi}{N+1}\right)\right)_{j=1}^{N}, \quad k=1, \ldots, N .
$$

The discrete iterative approximation of (4.12) takes the form

$$
f_{k}^{\delta}\left(x_{j}\right)=\left(I-\omega K_{h}\right)^{k} f_{0}\left(x_{j}\right)+\omega \sum_{i=0}^{k-1}\left(I-\omega K_{h}\right)^{i} g^{\delta}\left(x_{j}\right), \quad j=1, \ldots, N
$$

where $K_{h}=e^{-\sqrt{\mathbb{A}_{h}}}$ and $\omega<\frac{1}{\left\|K_{h}\right\|}=e^{\sqrt{\mu_{1}}}=2.7881$.

Figures 1-4, Table 1 show the comparisons between the exact solution and its computed approximations for different values $N, M$ and $\varepsilon$.

Figures 5-12, Table 2 show the comparisons between the exact solution and its computed approximations for different values $N, k, \omega$ and $\varepsilon$.

\section{Conclusion}

The numerical results (Figures 1-4) are quite satisfactory. Even with the noise level $\varepsilon=$ 0.01 , the numerical solutions are still in good agreement with the exact solution. In addition, the numerical results (Figures 5-12) are better for $(\omega=2.2305, \varepsilon=0.01)$ and $(\omega=1.9517, \varepsilon=0.001)$ and the other values are also acceptable.

In this study, a convergent and stable reconstruction of an unknown boundary condition has been obtained using two regularizing methods: truncation method and KozlovMaz'ya iteration method. Both theoretical and numerical studies have been provided. 

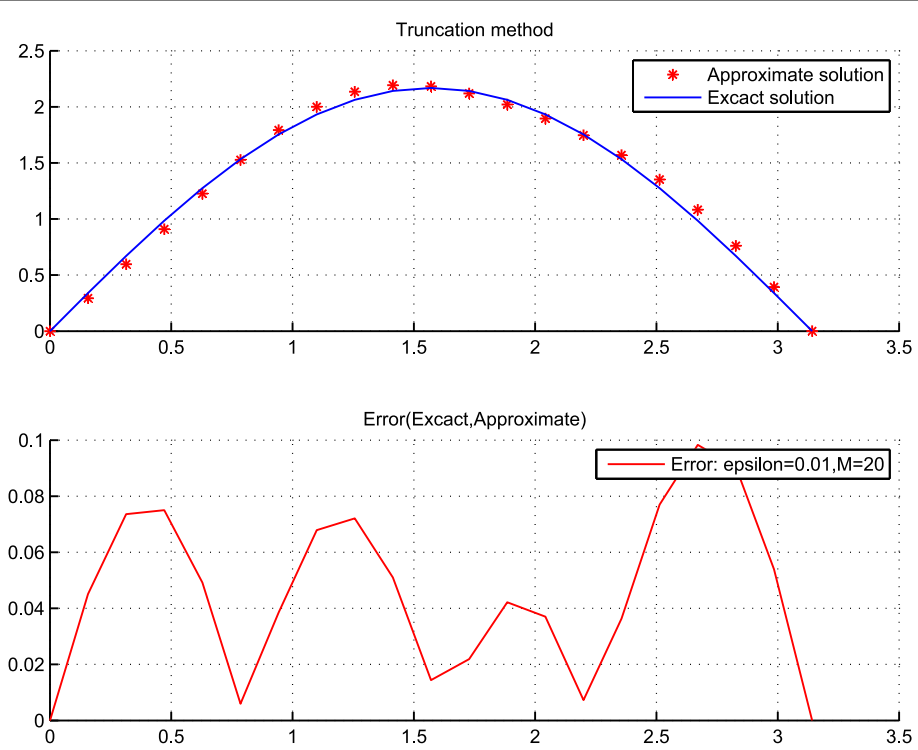

Figure $1 \mathrm{TM}$ with (noise level $=0.01$, truncation term $=4$, grid points of $T R=21$ ).
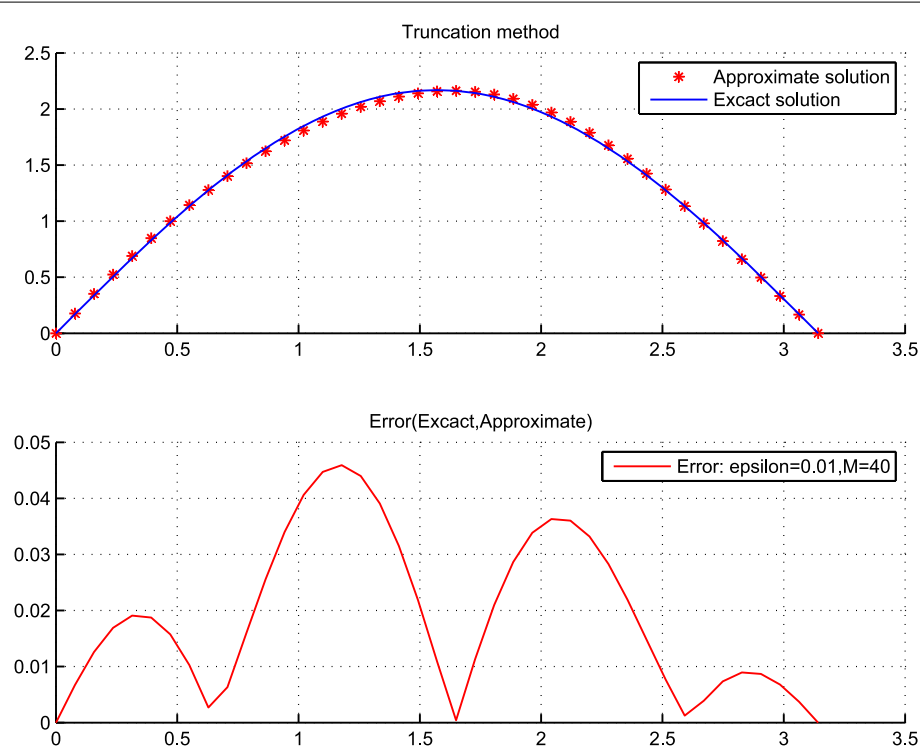

Figure $2 \mathrm{TM}$ with (noise level $=0.01$, truncation term $=4$, grid points of $T R=41$ ). 


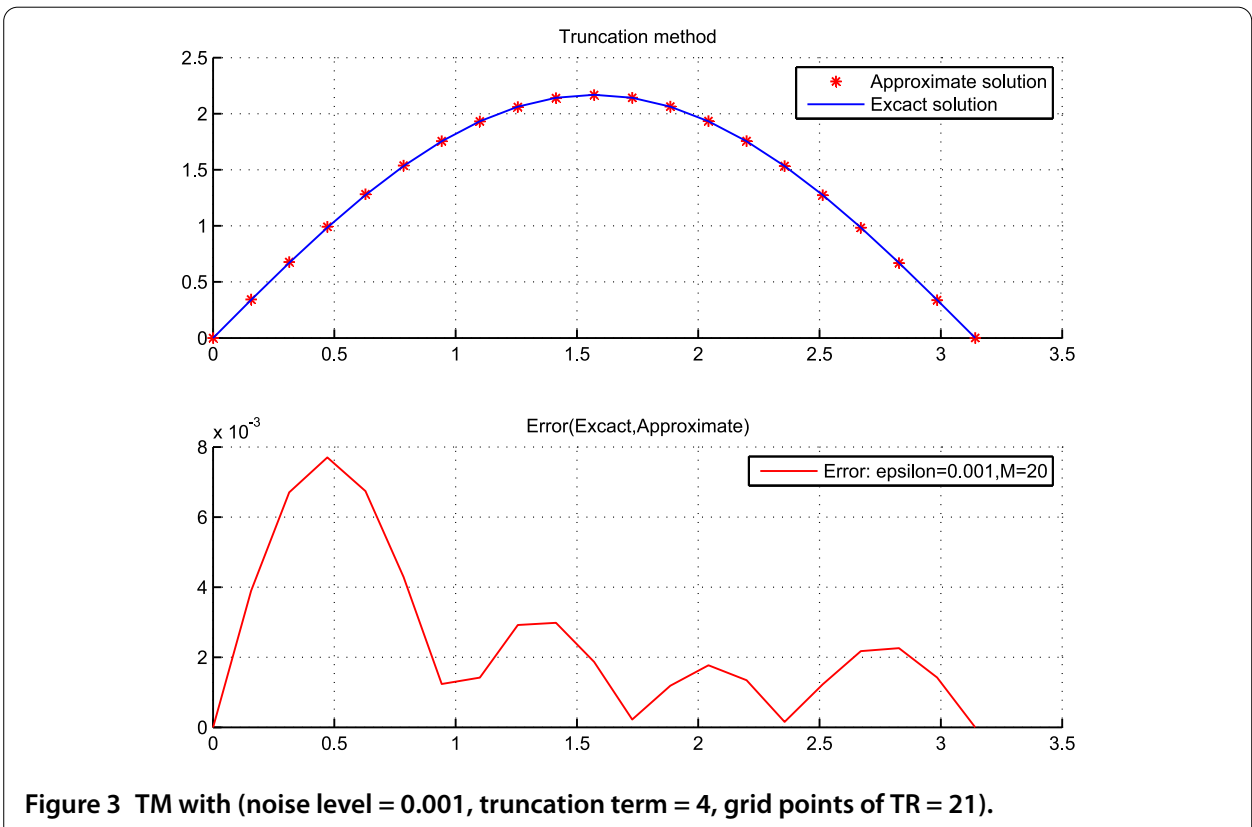

Figure $3 \mathrm{TM}$ with (noise level $=0.001$, truncation term $=4$, grid points of $T R=21$ ).
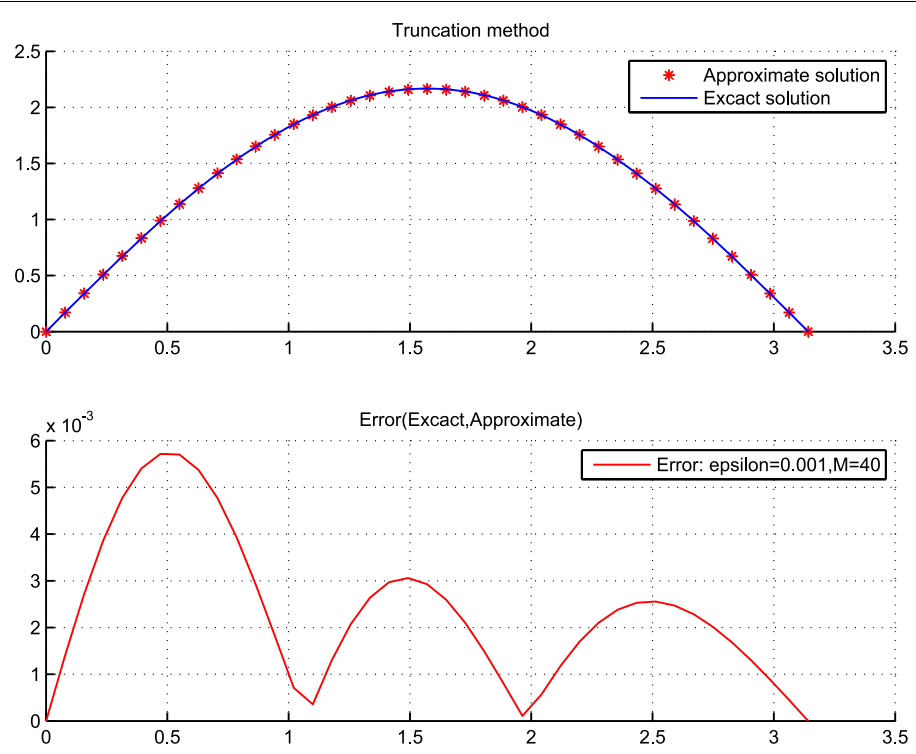

Figure $4 \mathrm{TM}$ with (noise level $=0.001$, truncation term $=4$, grid points of $\mathrm{TR}=41$ ).

Table 1 Truncation method: Relative error $E_{r}(f)$

\begin{tabular}{llll}
\hline $\boldsymbol{N}$ & $\boldsymbol{M}$ & $\boldsymbol{\epsilon}$ & $\boldsymbol{E}_{\boldsymbol{r}}(\boldsymbol{f})$ \\
\hline 20 & 4 & 0.01 & 0.0361 \\
40 & 4 & 0.01 & 0.0155 \\
20 & 4 & 0.001 & 0.0022 \\
40 & 4 & 0.001 & 0.0019 \\
\hline
\end{tabular}


Bouzitouna et al. Boundary Value Problems 2013, 2013:178

Page 19 of 23

http://www.boundaryvalueproblems.com/content/2013/1/178
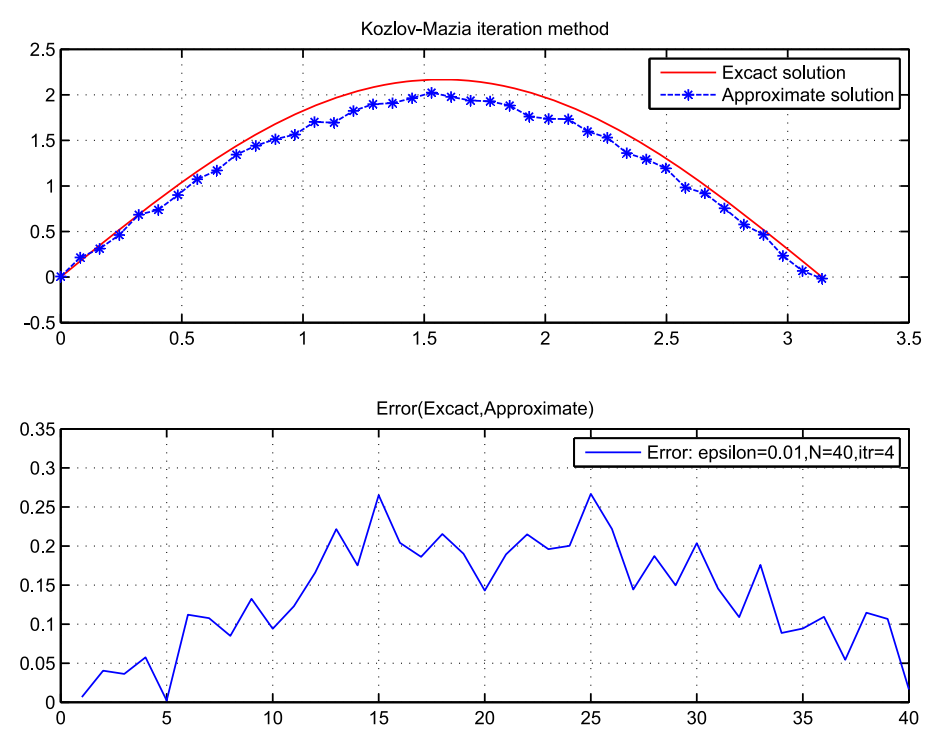

Figure $5 \omega=1.3941$.

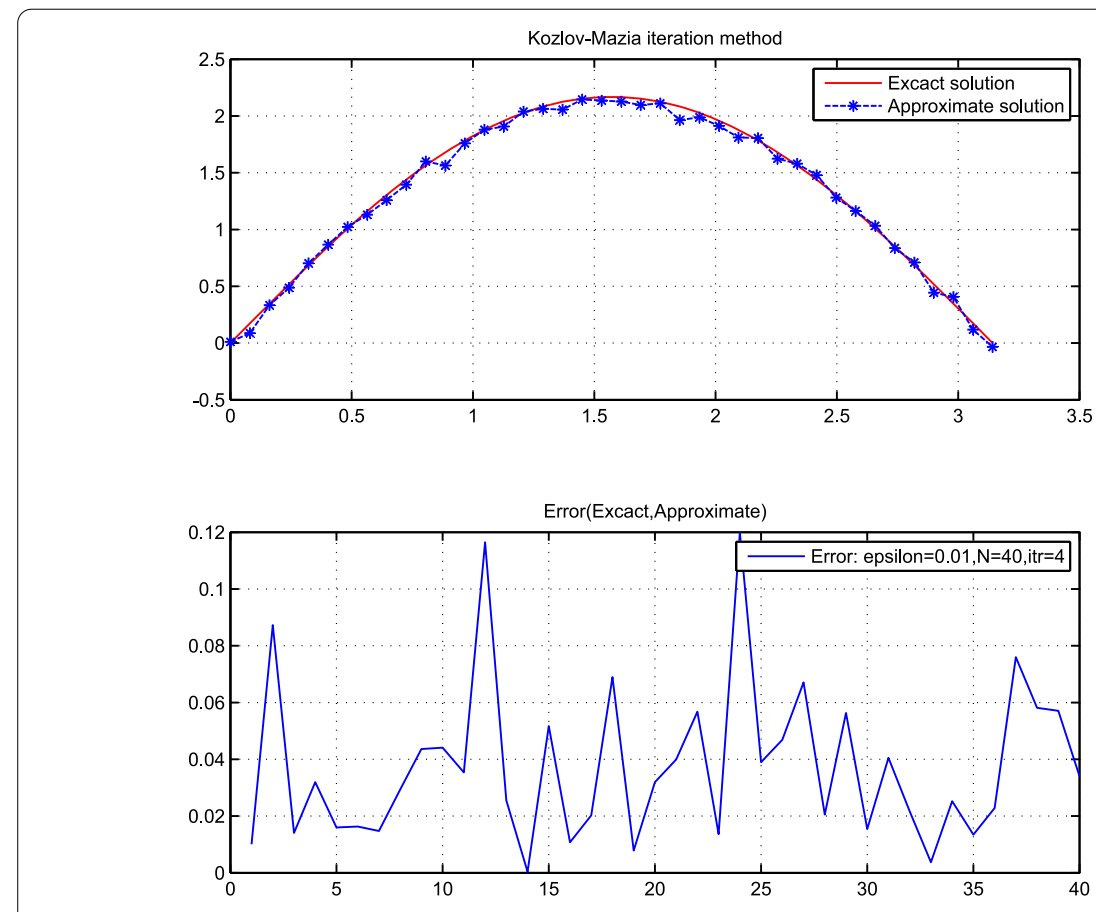

Figure $6 \omega=1.8587$. 
Bouzitouna et al. Boundary Value Problems 2013, 2013:178

Page 20 of 23

http://www.boundaryvalueproblems.com/content/2013/1/178
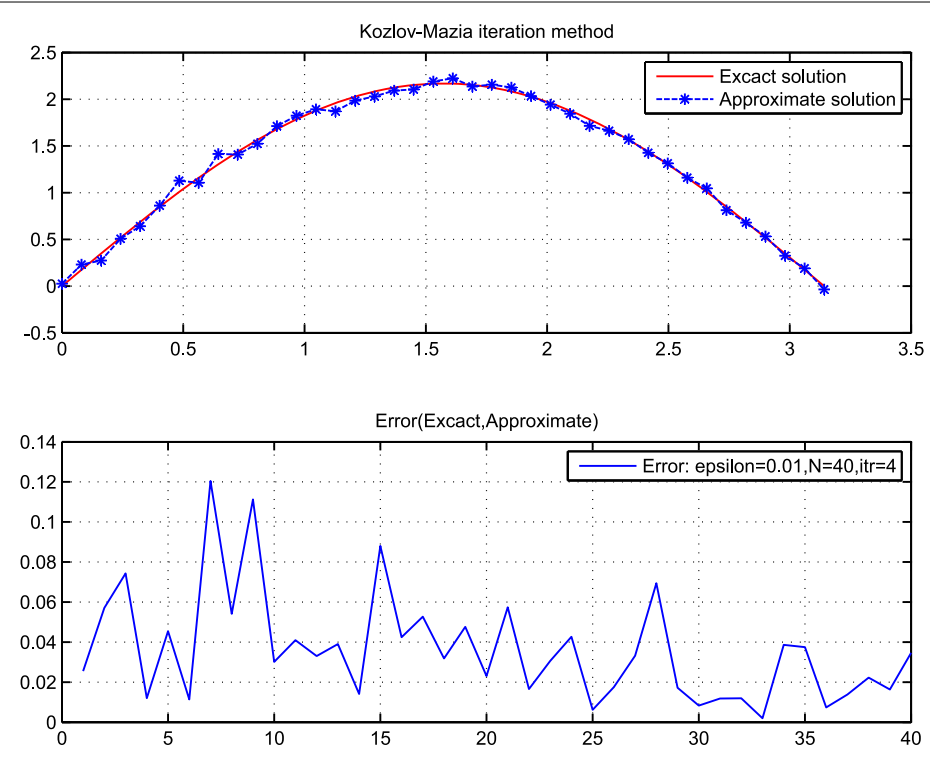

Figure $7 \omega=1.9517$.
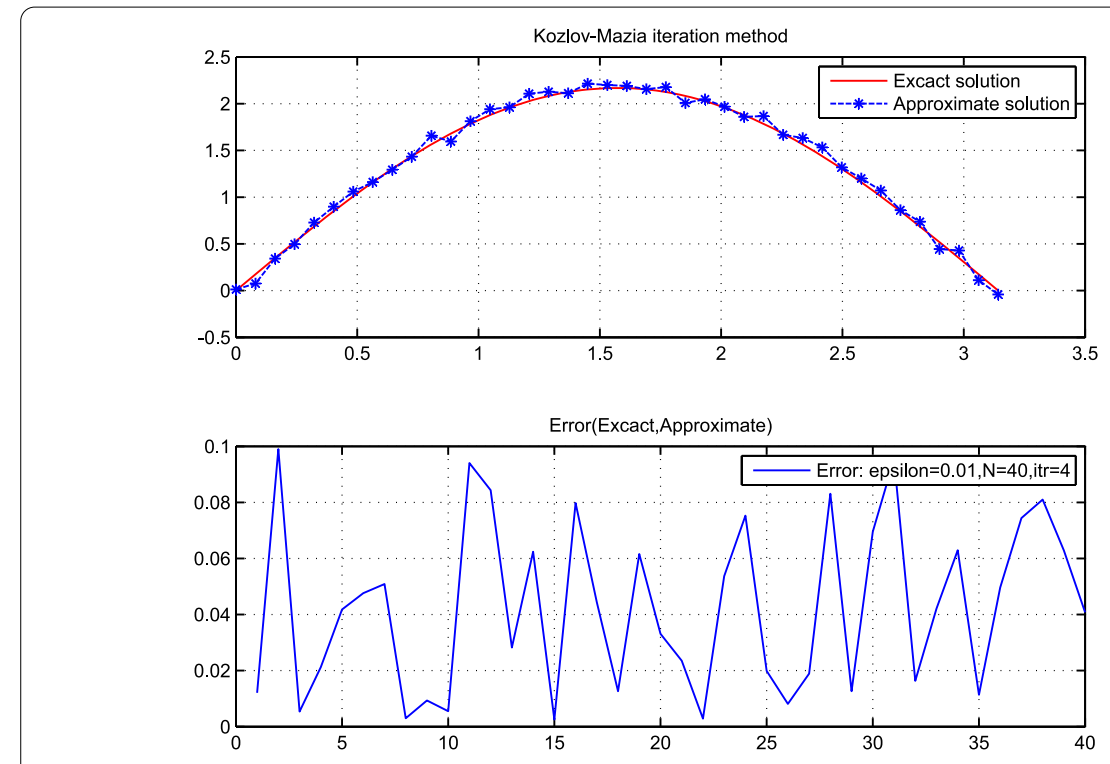

Figure $8 \omega=2.2305$. 

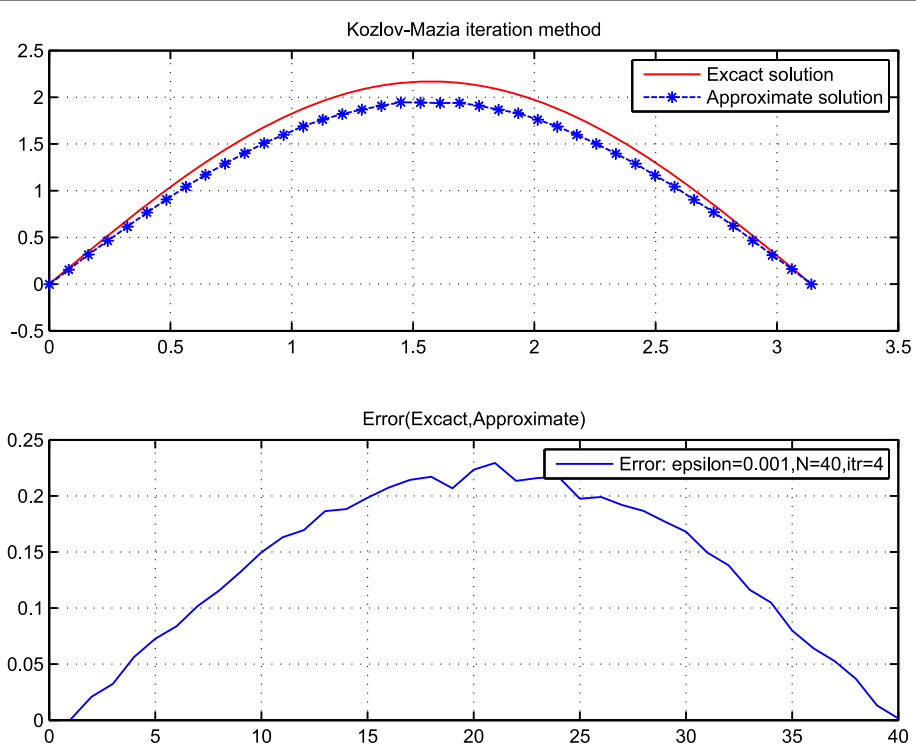

Figure $9 \omega=1.3941$.
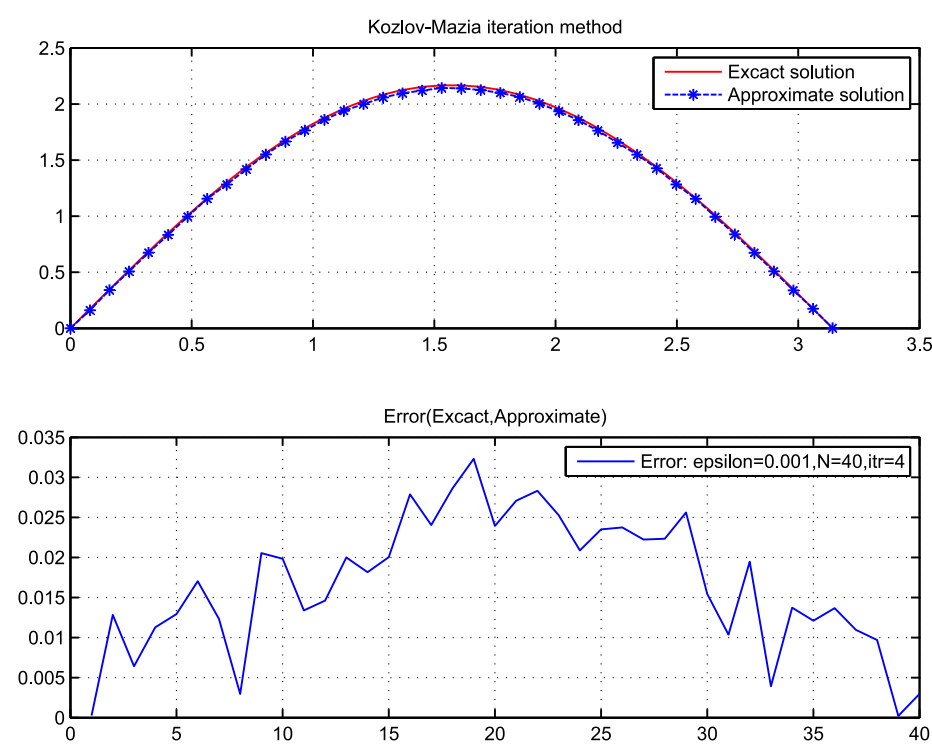

Figure $10 \omega=1.8587$. 


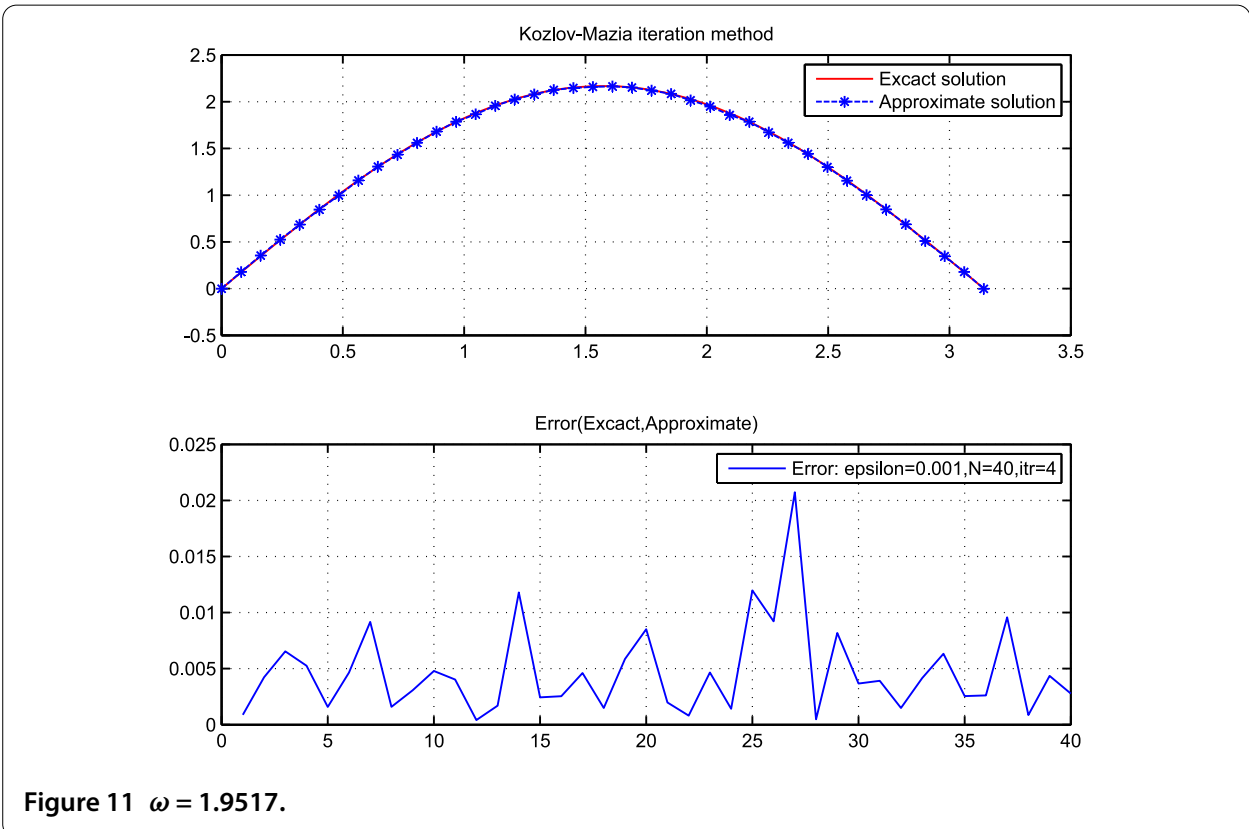

Figure $11 \omega=1.9517$
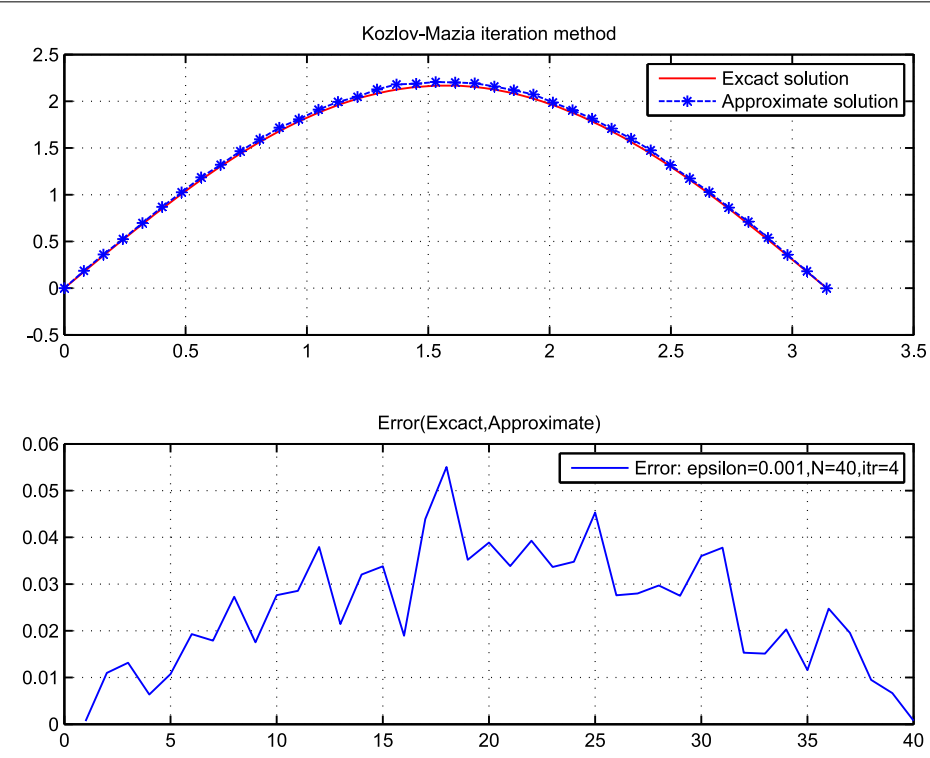

Figure $12 \omega=2.2305$

Table 2 Kozlov-Maz'ya method: Relative error $E_{r}(f)$

\begin{tabular}{lllll}
\hline $\boldsymbol{N}$ & $\boldsymbol{k}$ & $\boldsymbol{\epsilon}$ & $\boldsymbol{\omega}$ & $\boldsymbol{E}_{\boldsymbol{r}}(\boldsymbol{f})$ \\
\hline 40 & 4 & 0.01 & $0.5 \times 2.7881=1.3941$ & 0.0790 \\
40 & 4 & 0.01 & $2 / 3 \times 2.7881=1.8587$ & 0.0205 \\
40 & 4 & 0.01 & $0.7 \times 2.7881=1.9517$ & 0.0223 \\
40 & 4 & 0.01 & $0.8 \times 2.7881=2.2305$ & 0.0214 \\
40 & 4 & 0.001 & $0.5 \times 2.7881=1.3941$ & 0.0792 \\
40 & 4 & 0.001 & $2 / 3 \times 2.7881=1.8587$ & 0.0082 \\
40 & 4 & 0.001 & $0.7 \times 2.7881=1.9517$ & 0.0026 \\
\hline
\end{tabular}


Future work will involve the error effect arising in computing eigenfunctions and eigenvalues of the operator $A$ on the truncation method. The question is how to obtain some optimal balance between the accuracy of eigensystem and the noise level of input data.

\section{Competing interests}

The authors declare that they have no competing interests.

Authors' contributions

All authors have contributed equally. All authors read and approved the final manuscript.

\section{Author details}

${ }^{1}$ Applied Mathematics Laboratory, University Badji Mokhtar Annaba, P.O. Box 12, Annaba, 23000, Algeria. ${ }^{2}$ Department of Mathematics, 8 Mai 1945 Guelma University, P.O. Box 401, Guelma, 24000, Algeria.

\section{Acknowledgements}

The authors would like to thank the editor and the anonymous referees for their valuable comments and helpful suggestions that improved the quality of our paper. This work is supported by the DGRST of Algeria (PNR Project 2011-code: 8\u23\997)

\section{Received: 13 March 2013 Accepted: 18 July 2013 Published: 2 August 2013}

\section{References}

1. Cosner, C, Rundell, W: Extension of solutions of second order partial differential equations by the method of quasireversibility. Houst. J. Math. 10(3), 357-370 (1984)

2. Levine, HA, Vessella, S: Estimates and regularization for solutions of some ill-posed problems of elliptic and parabolic type. Rend. Circ. Mat. Palermo 34, 141-160 (1985)

3. Ivanov, DY: Inverse boundary value problem for an abstract elliptic equation. Differ. Equ. 36(4), $579-586$ (2000)

4. Dunford, N, Schwartz, J: Linear Operators, Part II. Wiley, New York (1967)

5. Pazy, A: Semigroups of Linear Operators and Application to Partial Differential Equations. Springer, New York (1983)

6. Brezis, H: Functional Analysis, Sobolev Spaces and Partial Differential Equations. Springer, New York (2011)

7. Prilepko, Al, Orlovsky, DG, Vasin, IA: Methods for Solving Inverse Problems in Mathematical Physics. Monographs and Textbooks in Pure and Applied Mathematics, vol. 222. Marcel Dekker, New York (2000)

8. Shlapunov, A: On iterations of non-negative operators and their applications to elliptic systems. Math. Nachr. 218 , 165-174 (2000)

9. Krasnosel'skii, MA, Vainikko, GM, Zabreiko, PP, Rutitskii, YB: Approximate Solutions of Operator Equations. Wolters-Noordhoff, Groningen (1972)

10. Krein, SG: Linear Differential Equations in Banach Space. Am. Math. Soc., Providence (1971)

11. Kozlov, VA, Maz'ya, VG: On iterative procedures for solving ill-posed boundary value problems that preserve differential equations. Leningr. Math. J. 1, 1207-1228 (1990)

12. Kozlov, VA, Maz'ya, VG, Fomin, AV: An iterative method for solving the Cauchy problem for elliptic equations. U.S.S.R. Comput. Math. Math. Phys. 31(1), 45-52 (1991)

13. Bastay, G: Iterative Methods for III-Posed Boundary Value Problems. Linköping Studies in Science and Technology, Dissertations No. 392. Linköping University, Linköping (1995)

14. Baumeister, J, Leitao, A: On iterative methods for solving ill-posed problems modeled by partial differential equations. J. Inverse III-Posed Probl. 9(1), 13-29 (2001)

15. Maxwell, D: Kozlov-Maz'ya iteration as a form of Landweber iteration (2011). arXiv:1107.2194v1 [math.AP] 12 Jul

16. Bakushinsky, AB, Kokurin, MY: Iterative Methods for Approximate Solution of Inverse Problems. Springer, Dordrecht (2004)

17. Hohage, T: Regularization of exponentially ill-posed problems. Numer. Funct. Anal. Optim. 21, $439-464$ (2000)

18. Deuflhardy, P, Engl, HW, Scherzer, O: A convergence analysis of iterative methods for the solution of nonlinear ill-posed problems under affinely invariant conditions. Inverse Probl. 14, 1081-1106 (1998)

19. Eldén, L, Simoncini, V: A numerical solution of a Cauchy problem for an elliptic equation by Krylov subspaces. Inverse Probl. 25, 065002 (22pp) (2009)

doi:10.1186/1687-2770-2013-178

Cite this article as: Bouzitouna et al.: Two regularization methods for a class of inverse boundary value problems of

elliptic type. Boundary Value Problems 2013 2013:178. 